\title{
Norois
}

Environnement, aménagement, société

222 | 2012

Xynthia

\section{La tempête Xynthia et la cartographie des « zones noires » sur le littoral français : analyse critique à partir de l'exemple de La Faute-sur-Mer (Vendée)}

The storm Xynthia and the cartography of the "black zones" on the French coast: a critical analysis from the example of the municipality of La Faute-surMer, Vendée department

Denis Mercier et Céline Chadenas

\section{OpenEdition}

\section{Journals}

Édition électronique

URL : https://journals.openedition.org/norois/3895

DOI : 10.4000/norois.3895

ISBN : 978-2-7535-1843-8

ISSN : $1760-8546$

Éditeur

Presses universitaires de Rennes

Édition imprimée

Date de publication : 28 février 2012

Pagination : 45-60

ISBN : 978-2-7535-1815-5

ISSN : 0029-182X

\section{Référence électronique}

Denis Mercier et Céline Chadenas, « La tempête Xynthia et la cartographie des « zones noires » sur le littoral français : analyse critique à partir de l'exemple de La Faute-sur-Mer (Vendée) », Norois [En ligne], 222 | 2012, mis en ligne le 30 mars 2014, consulté le 14 janvier 2022. URL : http:// journals.openedition.org/norois/3895 ; DOI : https://doi.org/10.4000/norois.3895 


PU⿴囗十⺝

\title{
La tempête Xynthia et la cartographie des «zones noires» sur le littoral français : analyse critique à partir de l'exemple de La Faute-sur-Mer (Vendée)
}

\author{
The storm Xynthia and the cartography of the "black zones" on the French coast: \\ a critical analysis from the example of the municipality of La Faute-sur-Mer, \\ Vendée department
}

\section{Denis Mercier* ${ }^{*}$ Céline Chadenas}

\footnotetext{
* Auteur correspondant : Tel : 33 (0)2 53487651 1. Fax : 33 (0)2 53487650 . Membre junior de l'Institut Universitaire de France

LETG-Géolittomer-Nantes - UMR 6554 CNRS, Université de Nantes, Campus du tertre, BP 81227,

-44312 NANTES Cedex 3, France (denis.mercier@univ-nantes.fr) (celine.chadenas@univ-nantes.fr)
}

Résumé : La tempête Xynthia du 28 février 2010 a été accompagnée d'une inondation de zones basses urbanisées du littoral atlantique français et a entraîné directement le décès de 41 personnes par submersion marine. Ces zones ont été qualifiées de « zone d'extrême danger », puis de «zone noire ». Une analyse sémantique est menée dans ce travail. Cette expression de «zone noire » introduit un nouveau seuil de dangerosité sur les cartes réalisées par les services de l'État. Les critères qui ont permis leur définition sont présentés et une analyse critique est réalisée en s'appuyant sur l'exemple de la commune de La Faute-sur-Mer en Vendée. Les habitations rachetées par l'État sont progressivement détruites sur ce territoire récemment urbanisé. Cette stratégie de repli est relativement nouvelle dans la politique française de gestion des territoires inondés et ne se fait pas sans difficulté. Réalisée dans l'urgence a posteriori après Xynthia, cette politique, dont l'objectif est d'empêcher de nouvelles victimes, devrait s'appliquer sur le territoire national a priori.

Abstract : On the $28^{\text {th }}$ of February 2010, the storm Xynthia flooded several low urbanized areas on the French Atlantic coast. This major marine flood directly caused the death of 41 people. These areas were first classified as "areas of extreme danger" and then as "black zones". A semantic analysis is conducted in this work as this expression of "black zone" introduces a new level of danger on the maps made by the State. This paper presents a critical analysis of the criteria that were used for these definitions and is applied to the town of La Faute-surMer in the département of Vendee, a recently urbanized territory. The houses located within the classified zones were first purchased by the State and are now gradually destroyed. This fallback strategy is relatively recent in the French management of flooded territories and is not without raising difficulties. Performed in emergency after Xynthia, this policy, which aims to prevent further victims, should always be implemented on the national territory without waiting for a disaster.

Mots clé : prévention - risque de submersion marine - inondation - vulnérabilité - aménagement littoral - Vendée

Keywords : prevention - marine submersion hazard - inundation - vulnerability - coastal management - Vendée 
"Le rôle principal y est tenu par le gouvernement même de la France, un gouvernement qui ne discernant qu'avec peine, en la matière dont il s'agit, un intérêt général sur lequel les gowvernés professent des opinions contradictoires, laisse souvent son action s'infléchir selon les vœux d'une minorité entreprenante ou n'ose opposer l'acte d'autorité aux préférences immédiates d'une majorité à courte vue. Un temps trop long s'écoule, entre les instants où se déchaînent les fureurs de la Loire, pour que les hommes soient tenus en haleine et disposés à la fermeté. La dernière catastrophe remonte à 1866. Il n'est donc plus possible de s'en faire une idée d'après les rapports des témoins oculaires. L'actuelle population du Val n'est plus informée du danger qui la menace que par une tradition dont la précision va s'émoussant d'année en année. Aussi n'est-elle point préparée à faire bon accueil au "prophète de malheur » qui viendrait lui recommander de faire un effort présent pour libérer l'avenir d'un risque. »

Roger Dion, 1961. Histoire des levées de la Loire, p. 248

Écrit il y a cinquante ans pour le risque d'inondation fluviale, cet extrait du travail de Roger Dion s'applique avec une actualité toute particulière pour le risque d'inondation par submersion marine. Le choix des gouvernants en matière sécuritaire et l'opposition des gouvernés ne font pas toujours bon ménage pour sauver des vies humaines. Les inondations des zones basses autour du Pertuis Breton consécutives à la tempête Xynthia nous le rappellent (figure 1). Cette dernière a fait 47 victimes en France, le 28 février 2010, dont 41 liées à la submersion marine (Chauveau et al., 2011a, 2011b), 29 morts en Vendée, tous sur la commune de La Faute-sur-Mer, et 12 en CharenteMaritime (Vinet et al., 2011a). Cependant, le bilan humain aurait pu être plus lourd sans la mobilisation des sapeurs-pompiers qui ont sauvé 1465 personnes de la noyade et de l'épuisement dans la nuit de l'événement. Suite à cette tragédie humaine, une nouveauté a été introduite dans l'approche de la réduction de la vulnérabilité de la société française face au risque d'inondation : le concept de «zone de danger mortel », très vite rebaptisé « zone noire », puis « zone de solidarité ». Dans la législation française et dans sa traduction cartographique dans les Plans de Prévention des Risques (PPR), ce concept se traduit par un nouveau seuil. Prévue pour éviter que de nouvelles victimes succombent à des inondations futures, par submersion marine ou par débordement des cours d'eau, cette approche devrait s'appliquer $a$ priori et non a posteriori sur le territoire. En France métropolitaine, au-delà du seul risque d'inondation, les zones de danger mortel concernent aussi les zones avalancheuses par exemple. Cette approche initiée avec grande difficulté sur le littoral atlantique dans les mois qui ont suivi la tempête Xynthia, n’a pas été appliquée suite aux inondations meurtrières (25 morts) provoquées par des pluies torrentielles les 14 et 15 juin 2010 dans le Var (Vinet et al., 2011b). Cette nouvelle approche dans la politique de réduction de la vulnérabilité potentielle pose une série de questions : est-elle réalisable politiquement et acceptable humainement par la société?; comment peuton définir des zones de danger mortel?; sur quels critères?; comment peut se traduire cartographiquement une probabilité de mortalité? ; est-il possible de faire une carte de France à l'échelle communale et infracommunale des «zones noires » potentielles?; si cette cartographie est réalisée, elle implique un repli stratégique, c'est-à-dire de détruire les maisons situées en «zone noire » : comment faire accepter ce phénomène nouveau à la population (il a débuté à La Faute-sur-Mer à la fin du mois de mars 2011)?

Cette étude propose de revenir sur l'évolution sémantique du concept de "zones noires ", de mener une analyse critique des critères qui permettent de les définir en prenant l'exemple de la commune de La Faute-sur-Mer située à l'embouchure de l'estuaire du Lay dans le sud du département de la Vendée (figure 2).

\section{ZONES DE DANGER MORTEL, ZONES NOIRES, ZONES DE SOLIDARITÉ : ÉVOLUTION SÉMANTIQUE}

La tempête Xynthia a fait l'objet d'une couverture médiatique et de réactions politiques sans précédent dans l'histoire des risques naturels en France. Ces dernières ont été nombreuses et rapides. L'espace inondé a fait l'objet de plusieurs dénominations au cours des semaines et des mois qui ont suivi l'événe- 

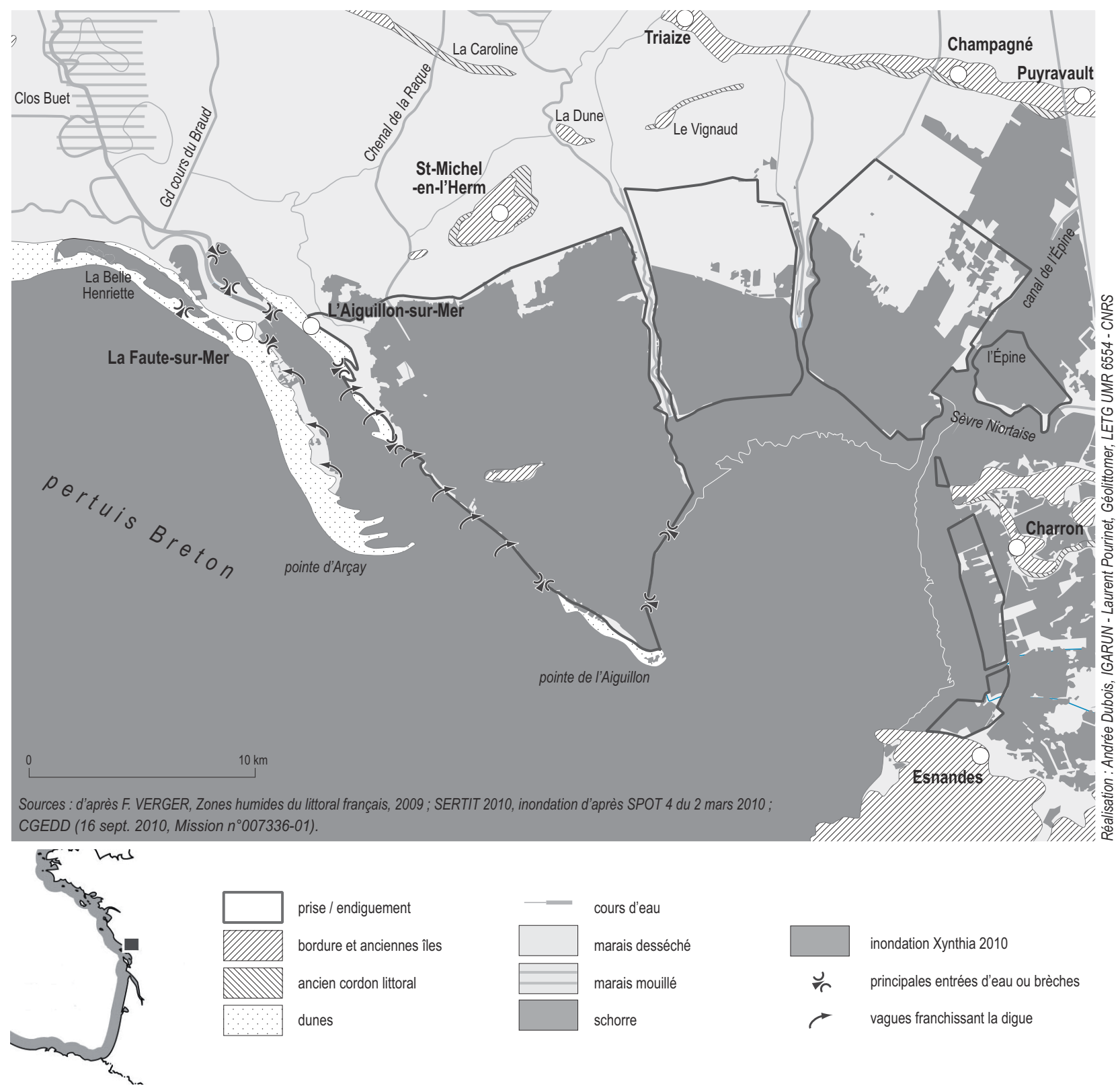

Figure 1 : Localisation de la zone d'étude Study area

ment météo-marin, dont le terme critiqué de «zone noire » (figure 3).

\section{Zones de « danger mortel »}

Dès le $1^{\text {er }}$ mars 2010 , soit le lendemain de la catastrophe, le Président de la République française est venu sur le littoral atlantique constater les dégâts. De retour deux semaines plus tard, dans son discours du 16 mars 2010 à La Roche-sur-Yon, le Président a déclaré : «Dans les zones exposées aux plus forts risques, nous n'avons pas le droit de laisser nos concitoyens se réinstaller comme si de rien n'était. Ce serait irresponsable. [...] Une cartographie précise est en cours d'élaboration destinée à identifier les sites à fort aléa. Mais je veux être clair sur ce point : on ne 


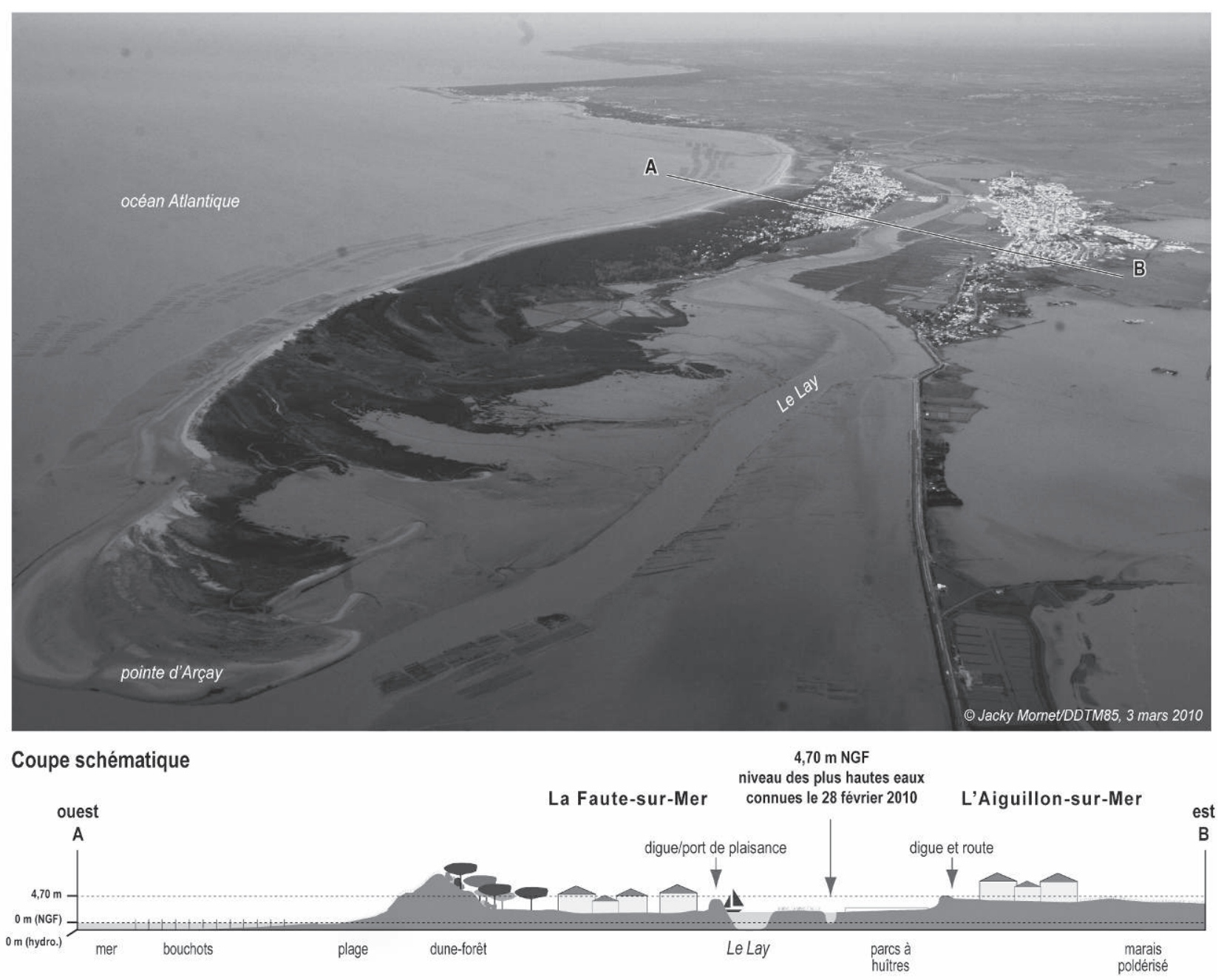

Figure 2 : Vue aérienne oblique et coupe d'interprétation de l'estuaire du Lay Aerial oblic view and landscape interpretation of the estuary of Lay

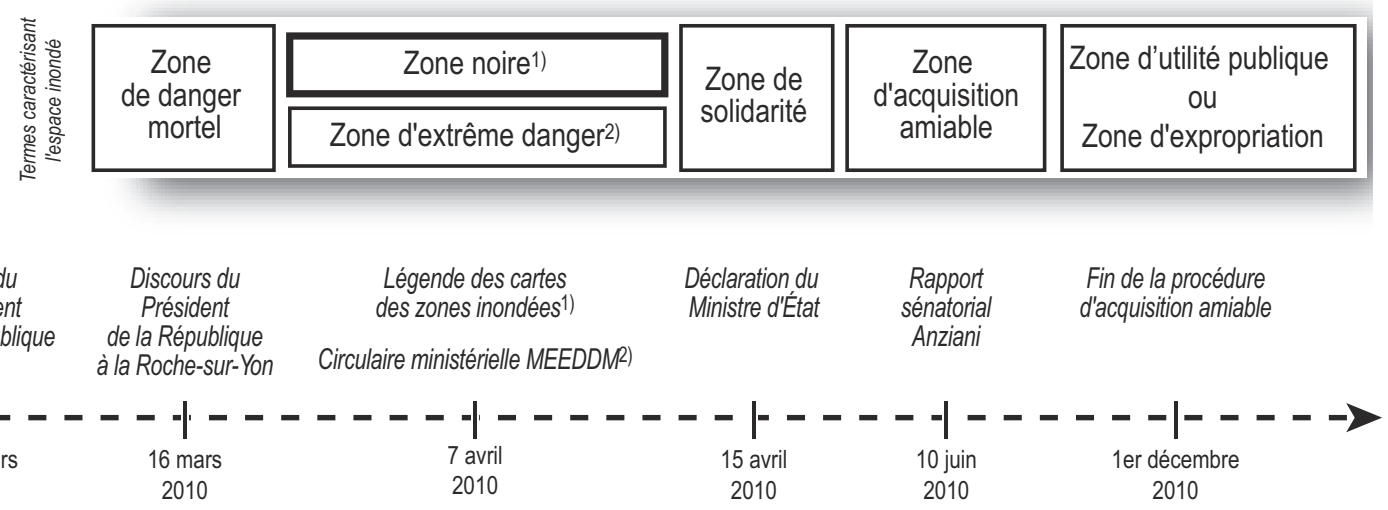

Figure 3 : Évolution sémantique du concept de «zone noire» Semantic evolution of the "black zone" concept 
négociera pas l'aléa, il s’impose à tous, l'aléa n’est pas à géométrie variable. [...] Là où il y a risque mortel, personne ne reviendra y habiter. Dans ces zones, il est indispensable que toutes les reconstructions et tous les aménagements soumis à permis de construire soient désormais interdits. [...] Enfin, je veux dire ma conviction, qu'après le 28 février 2010, rien ne doit plus être comme avant en matière de prévention des catastrophes naturelles. [...] Nous n'autoriserons aucune reconstruction en zone mortelle. [...] Naturellement, il ne s'agit pas de passer d'un excès à l'autre et de condamner la totalité du littoral français. Il s'agit de faire la différence entre des zones mortelles et des zones où il $\mathrm{y}$ a un risque faible de petites inondations ».

Il est précisé que ces zones sont déclarées inhabitables car exposées à un danger mortel, « c'est-à-dire à un risque naturel tel qu'il n'est pas possible d'y mettre en place une protection efficace des populations » (Anziani, 2010a).

Cette appellation de «zones de danger mortel » est remplacée par celle de "zones à risque d'extrême danger pour la vie humaine » dans la circulaire ministérielle du MEEDDM du 7 avril 2010 relative aux mesures à prendre suite à la tempête Xynthia du 28 février 2010.

\section{«Zones noires »}

Ces zones ont été baptisées « zones noires » dans la presse à la fin du mois de mars (La Gazette des communes du 29 mars 2010) dans l'attente des réunions publiques des 7 et 8 avril 2010 de présentation des zonages des secteurs inondés. Sur ces cartes, la couleur noire fait son apparition et désigne les « zones d'extrême danger à délocaliser ». Dès le 7 avril, dans le compte rendu du conseil des ministres, il est écrit : " Dans les zones d'extrême danger, où les risques peuvent être mortels, il n'est pas acceptable de laisser les habitants se réinstaller. Ces zones, dites "zones noires", doivent retourner à l'état naturel, avec d'éventuelles activités diurnes, comme par exemple les activités ostréicoles. » Cette appellation de «zone noire » est donc d'abord une dénomination officielle, largement relayée ensuite par la presse à partir des réunions des 7 et 8 avril 2010. L'attribution du noir se comprend dans la logique sémiologique des cartes des PPR avec une graduation des couleurs (bleu, rouge, noir). Par ailleurs, ce choix a marqué les esprits de la population, le noir étant associé à la mort et au deuil.

Dans les zonages des plans de prévention des risques, l'attribution d'une nouvelle couleur se justifie aussi puisqu'il existe, depuis la loi Barnier du 2 février 1995, des zones en bleu clair, en bleu foncé, en rouge.

Cependant, la gamme chromatique s'est progressivement étendue puisqu'à ces couleurs ont été ajoutés le jaune, le orange et le violet parfois (c'est le cas dans l'Isère, Douvinet et al., 2011). Les «zones jaunes » ou «zones de prescription », sont « des zones où le risque de submersion marine, bien que réel et fort, peut être maîtrisé en imposant aux habitants d'effectuer des aménagements spécifiques sur leur résidence (aménagement d'un étage refuge par exemple). Les « zones oranges », catégorie hybride, définissant des zones dans lesquelles des expertises supplémentaires devaient être réalisées afin de déterminer si elles ressortissaient de la «zone noire » ou de la « zone jaune », ont été délimitées » (Anziani, 2010b). Les « zones violettes » sont des zones constructibles où l'aléa est moyen. Pour éviter tout principe de constructibilité inconditionnelle, deux zones ont été créées. Une zone «violet dur» inconstructible en l'état, qui peut le devenir après études et travaux, à conditions que les études n'infirment pas cette possibilité. En cas d'ouverture à la constructibilité, le PPRI doit être révisé. Une zone «violet mou » correspond à une « zone admise » à la construction avec des travaux sous la maîtrise d'ouvrage collective. Le principe des « zones violettes » revient à légitimer les constructions dans les zones où l'aléa est connu. Depuis Xynthia, cette pratique des «zones violettes » a été arrêtée (Douvinet et al., 2011).

\section{«Zones de solidarité » ou «zones d'acquisition amiable »}

Pour tenter une diversion sémantique à la polémique qui s'amplifiait alors sur la délimitation de ces espaces, les zones noires sont rebaptisées « zones de solidarité » le 15 avril 2010 par le ministre d'État Jean-Louis Borloo, lors d'une visite dans les zones sinistrées.

L'évolution sémantique traduit les hésitations des politiques. Leur seule certitude est de vouloir rassurer les populations quant aux indemnisations qui les touchent. Une autre appellation le prouve. Le 
rapport d'information du Sénat $n^{\circ} 554$ de M. Alain Anziani déposé le 10 juin 2010 propose de « retenir le terme de "zone d'acquisition amiable" plutôt que celui de "zone noire" ou de "zone de solidarité", afin de souligner que la principale caractéristique de ces zones est qu'une procédure d'indemnisation rapide et équitable y sera menée ».

Cette confusion dans l'expression publique a été un facteur d’inquiétude qui a engendré des réactions vigoureuses d'une partie des populations sinistrées. Elle a contribué à entretenir des incertitudes sur le statut juridique des «zones noires ». Ce sentiment a été exacerbé par le manque de transparence du processus de délimitation des zones à risque. En témoigne le refus des préfectures de communiquer aux associations de victimes les études sur lesquelles les experts s'étaient appuyés pour délimiter les « zones de danger mortel ", et la formation subséquente d'un recours en référé (Anziani, 2010a).

"La décision de mettre en place un tel zonage transparaît dès le discours du Président de la République du 16 mars 2010 et elle est matérialisée le 18 mars 2010, dans une circulaire demandant aux préfets de Vendée et de Charente-Maritime de procéder, en lien étroit avec les cabinets ministériels et avec l'aide d'experts nationaux, à la délimitation des "zones d'extrême danger". » (Anziani, 2010b.)

\section{«Zones d'utilité publique» ou « zones d'expropriation »}

Depuis le $1^{\text {er }}$ décembre 2010, la zone de solidarité est devenue "Zone d'Utilité Publique » ou "Zone d'Expropriation ». La zone de solidarité définie en avril dernier, qui permet à chaque habitant de bénéficier, s'il le souhaite d'un engagement de l'État d'acquérir son bien est maintenu. Cependant, pour les parcelles occupées par des habitations n'ayant pas fait l'objet d'une acquisition amiable, elles seront soumises à la déclaration d'utilité publique en vue d'une expropriation.

Dans tous les cas de figures, pour cartographier le danger de mort suite à des inondations, il est nécessaire de définir des critères de dangerosité.

\section{LES CRITÈRES DE DÉFINITION DES «ZONES NOIRES »: ANALYSE CRITIQUE}

Les critères permettant de déterminer les « zones d'extrême danger » sont définis par la circulaire ministérielle du 18 mars 2010 mettant en évidence les critères de dangerosité. Plusieurs critères ont été établis et les zones remplissant deux de ces critères ont été intégrées aux zones noires ${ }^{1}$.

Le premier d'entre eux situe le niveau d'eau à $1 \mathrm{~m}$. C'est la « référence en raison de la dangerosité pour tout individu même sportif » (Anziani, 2010b).

Le deuxième critère correspond à « une distance comprise entre 90 et 110 mètres derrière un ouvrage de protection qui constitue également une zone de survitesse forte en cas de rupture de l'ouvrage dans laquelle il faut éviter toute urbanisation » (Conseil Général de Charente-Maritime, 2010; Anziani, 2010b).

Le troisième critère correspond à la cinétique de submersion lorsqu'elle a présenté un danger pour les personnes. Il est spécifié dans le rapport Pitié et Puech (2010) : «On considère habituellement comme dangereuse une montée des eaux de $1 \mathrm{~m}$ en moins d'une demi-heure : lorsque cela se produit les personnes surprises pendant leur sommeil ne prennent conscience de la situation que lorsque le niveau est relativement élevé et peuvent avoir des difficultés à gagner un refuge même proche. »

Le quatrième critère concerne les habitations très endommagées et ne pouvant que difficilement être reconstruites avec un refuge.

Enfin, le cinquième et dernier critère considère que la zone doit former un ensemble cohérent et homogène, et ne crée pas de mitage urbain. En effet, le maintien de propriétés éparses les fragiliserait, justement à cause de leur isolement, et rendrait leur évacuation plus difficile en cas de sinistre (Anziani, 2010a).

Quatre critères parmi ces cinq sont $a$ priori faciles à traduire en termes de cartographie (figure 4). Le troisième, correspondant à la vitesse de submersion, l'est déjà beaucoup moins. Cependant, le passage de la théorie à la pratique, même avec des critères bien identifiés, est loin d'être évident.

1. Depuis la soumission de cet article le 23 mai 2011, une circulaire du MEDDTL en date du 27 juillet 2011 (NOR : DEVP1119962C) précise les critères de prise en compte du risque de submersion marine dans les plans de prévention des risques naturels littoraux. 


\section{Critère $n^{\circ} 1$ : hauteur d'inondation $>1 \mathrm{~m}$}

a - différentiel de dangerosité en fonction de l'âge et de l'état de santé des habitants

b - différentiel de dangerosité en fonction de l'architecture des maisons (plain-pied, pièce de survie à l'étage...)

c - différentiel de dangerosité en fonction de la microtopographie

$b$

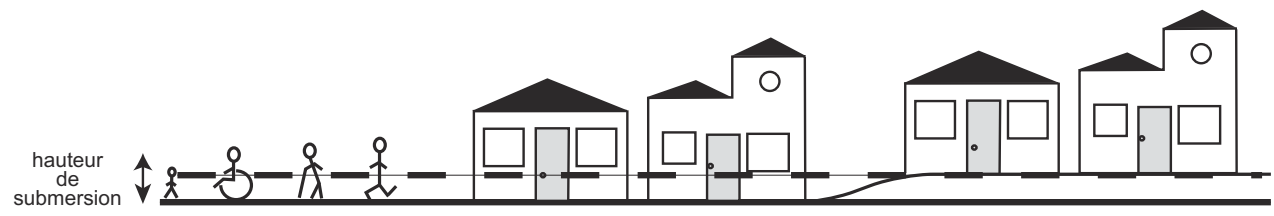

Critère $n^{\circ} 2$ : distance de $100 \mathrm{~m}$ par rapport à une digue

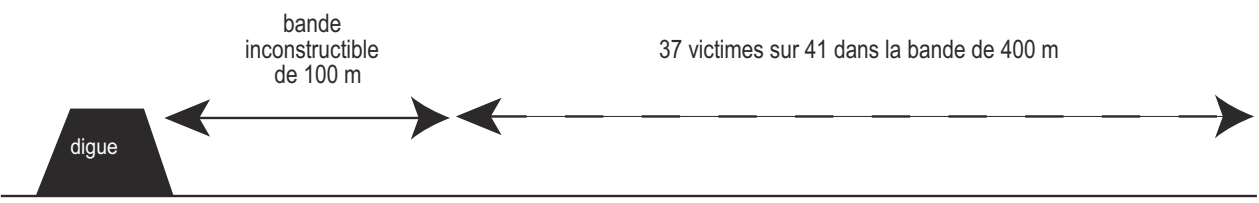

Critère $n^{\circ} 3$ : cinétique de submersion (+ $1 m$ en $1 / 2$ heure)

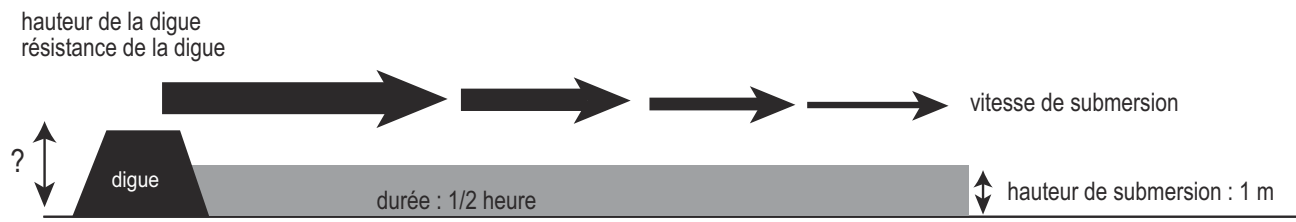

Critère $n^{\circ} 4$ : résistance des bâtiments à l'inondation

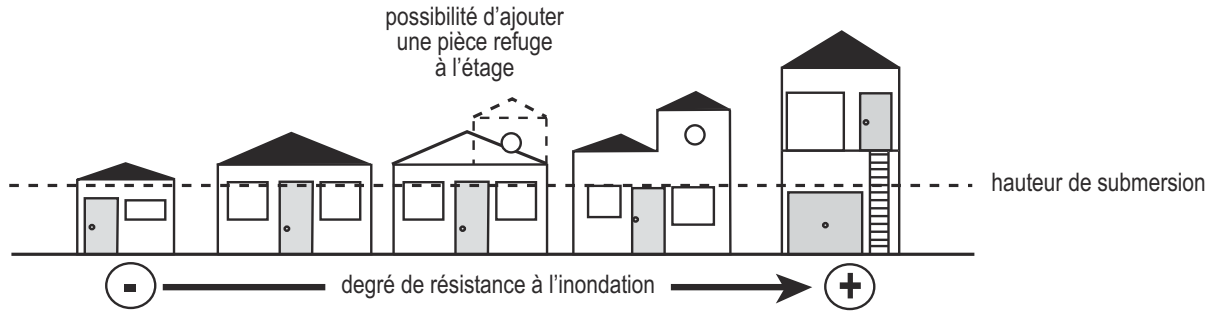

Critère $n^{\circ} 5$ : ensemble cohérent et homogène pour éviter le mitage
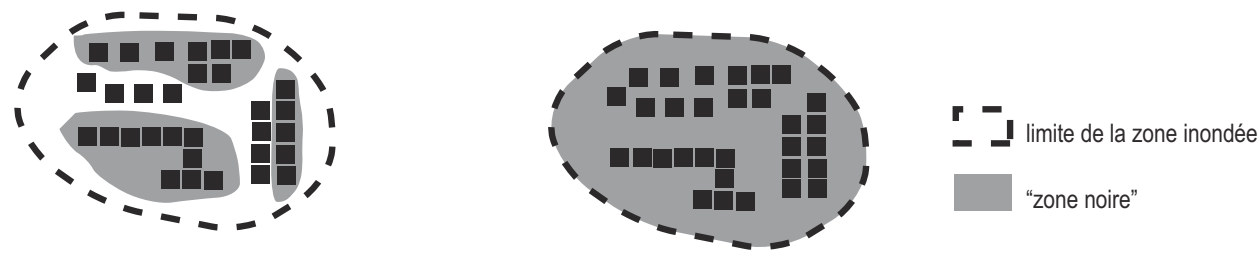

Figure 4 : Critères de définition des "zones noires »

Criteria of definition of the "black zones" 
Le premier critère relatif à la hauteur d'un mètre de submersion est le plus facile à cartographier. Il s'est appuyé sur les relevés des laisses de mer sur les communes touchées par la tempête Xynthia effectués par les services de l'État dès le $1^{\text {er }}$ mars. Cependant, les zones littorales urbanisées sont souvent des zones d'accrétion sédimentaire générée par le vent, la houle, la dérive littorale. Ces dynamiques créent une microtopographie chaotique ce qui conduit à une parcellisation de l'espace. Le simple critère altimétrique d'un mètre de hauteur d'eau est donc appliquable sur le terrain au cas par cas, mais conduit à un mitage de l'espace. Des maisons voisines de quelques mètres, parfois seulement séparées par une route ou un chemin, peuvent alors se retrouver dans une zone mortifère ou pas (figure 5). Par ailleurs, cette hauteur de 1 mètre retenue correspond à la « limite de déplacement debout d'un adulte sportif stressé » selon les termes du MEEDDM. Cependant, la hauteur dangereuse pour une personne à mobilité réduite, une personne âgée ou un enfant est inférieure à un mètre. Alors pourquoi ne pas avoir utilisé la hauteur la plus faible,

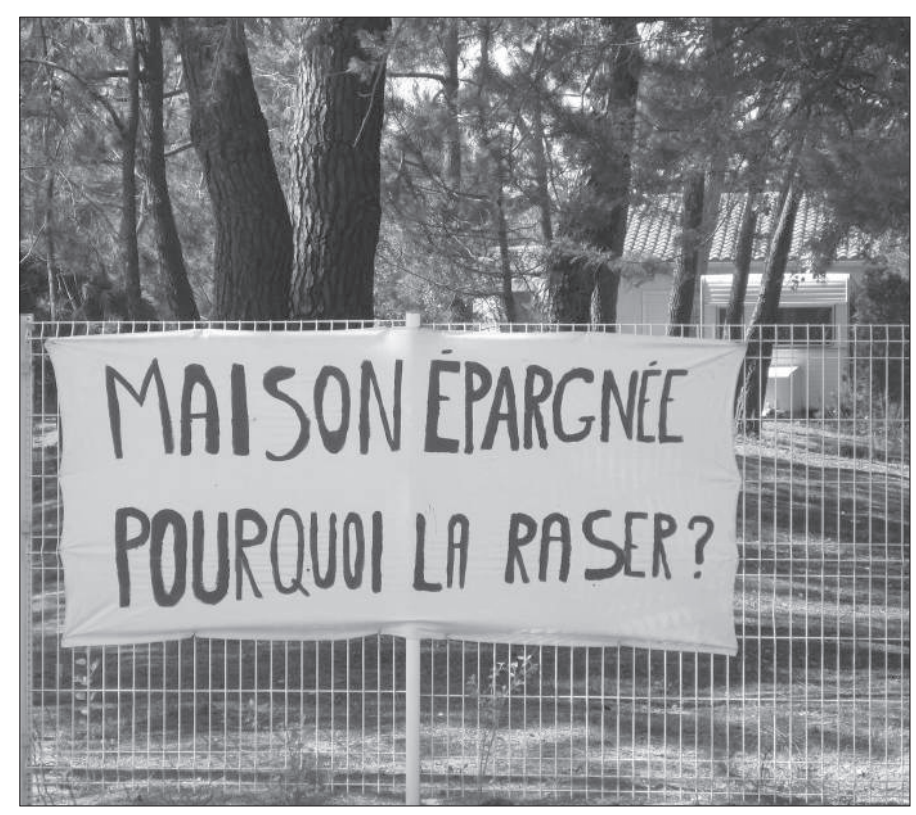

Figure 5 : Maison épargnée par l'inondation sur la commune de La Faute-surMer se situant pourtant dans la « zone noire ». Elle a été construite sur une accrétion sédimentaire la plaçant en hauteur par rapport à la zone inondée, d'où le questionnement de ses propriétaires (cliché D. Mercier, 20 mai 2010).

House saved by the flood on the municipality of La Faute-surMer being nevertheless situated in the "black zone". It was built on a sedimentary accretion placing it in height with regard to the flooded zone, where from the questioning of its owners. qui permettrait probablement de réduire le potentiel de mortalité? Les statistiques montrent qu'il y a une surreprésentation des plus de 60 ans sur les littoraux français (selon l'Observatoire du littoral du MEDDTL, en 1999, 25,4\% des habitants avaient plus de 60 ans contre $21,3 \%$ pour la moyenne française). Les travaux de Vinet et al. (2011b) ont montré la corrélation entre hauteur d'eau et âges des victimes lors des inondations consécutives à la tempête Xynthia. En outre, la logique d'un mètre s'applique sur l'altitude de l'entrée de la maison et ne tient pas compte d'un différentiel possible entre des maisons de plain-pied et des maisons construites sur plusieurs niveaux. Ce seul critère de hauteur d'un mètre n'est pas applicable à l'ensemble du territoire national. En effet, bien des secteurs inondables sont submergés sur des hauteurs plus importantes (2 à $4 \mathrm{~m}$ ) sans qu'il y ait mise en danger de la vie des personnes. C'est le cas des zones inondables des bords de cours d'eau comme la Loire (Jousseaume et Mercier, 2009; Vinet, 2010).

Le deuxième critère de distance de 100 m par rapport à une digue est également critiquable sur plusieurs points. Faut-il inclure aussi les édifices naturels comme les dunes? En certains endroits, la protection qu'elles semblent offrir ne résiste pas à une tempête. Ces accumulations sédimentaires peuvent aussi se rompre face à l'assaut des vagues, comme ce fut le cas le 28 février 2010 au lieu-dit la Belle-Henriette sur la commune de La Faute-surMer où la dune s'est rompue là où autrefois l'estuaire du Lay se jetait dans l'océan. Ces constructions sédimentaires constituent des remparts de protection, mais peuvent également être fragilisées par le piétinement créé par des chemins d'accès à la mer, abaissant parfois la hauteur de la dune de 2 à 3 mètres, facilitant ainsi le passage de la mer en cas de tempête. Pourquoi la distance de $100 \mathrm{~m}$ ? Est-on hors de danger mortel à $120 \mathrm{~m}$ ou $200 \mathrm{~m}$ de la digue lorsque celle-ci est submergée ou éventrée? Toutes les digues constituent des pièges pour les habitants qui pensent être en sécurité derrière ses ouvrages. Pour Xynthia, 37 des 41 victimes décédées en Charente-Maritime et en Vendée se trouvaient à moins de $400 \mathrm{~m}$ derrière une digue (Vinet et al., 2011 b). Dans le rapport de Pitié et Puech (2011) il est écrit : «En cas de rupture de digue ou de cordon dunaire, jusqu'à une distance de $400 \mathrm{~m}$ de la brèche, le débit devient très important en quelques 
minutes et les courants sont violents à proximité de la brèche. " Par ailleurs, la distance de 100 mètres égale partout en France interroge car la dangerosité dépend surtout de la hauteur de la digue, qui elle-même, introduit des vitesses de propagation de la submersion différentes, évoquée par le troisième critère de la cinétique de l'inondation.

Le critère lié à la cinétique de l'inondation est prépondérant : « Risque de caractère brutal en particulier par suite de présence de digues » (Pitié et Puech, 2010). Cette dernière précision est intéressante car elle reconnait implicitement que les digues aggravent le caractère dangereux d'une inondation en renforçant la vitesse de propagation de l'onde de submersion. Ce principe est connu depuis plusieurs siècles par les ingénieurs. Les débits ont été estimés à $400 \mathrm{~m}^{3} / \mathrm{s}$ au droit de la digue de La Faute-sur-Mer. Selon les lois des seuils déversant, le débit est :

$$
Q=\mu \operatorname{Lh}^{3 / 2} \sqrt{2} g
$$

où $\mu=$ coefficient de forme pris ici à 0,32 pour un seuil non profilé, $\mathrm{L}=$ la longueur déversante à pleine épaisseur de $800 \mathrm{~m}, \mathrm{~h}=$ la hauteur d'eau devant le seuil soit $0,40 \mathrm{~m}$ en moyenne, $\mathrm{g}=$ la pesanteur, soit $9,81 \mathrm{~m} / \mathrm{s}^{2}$

Cependant, la vitesse de submersion est très difficile à calculer. Le plus souvent, les valeurs prédites issues des modélisations mathématiques sont très différentes des valeurs déduites d'observations effectuées sur des ruptures réelles ou simulées d'ouvrages (Seidou et al., 2004 ; Zerrouk et Marche, 2005). En effet, le paramètre le plus important est le mode de rupture de l'ouvrage soit par surverse, soit par des brèches dans l'ouvrage. Il en est de même avec la vitesse de montée des eaux dans les secteurs habités. En effet, celle-ci dépend, entre autres, de la capacité de la digue à résister face à l'assaut des vagues. En cas de rupture de l'ouvrage, les vitesses de l'onde de submersion sont considérables. Ce paramètre est certainement le plus important pour la survie des populations résidant derrière une digue, mais aussi le plus aléatoire dans le temps et l'espace et le plus difficile à intégrer dans la cartographie préventive.

Par ailleurs, elle signifie aussi que plus les digues sont hautes, donc plus on surélève celles-ci, plus la dangerosité de la digue est importante. Non seulement, les digues introduisent un sentiment erroné de sécurité pour les personnes qui vivent derrière ces protections illusoires, mais en plus elles aggravent potentiellement le risque mortel. Les digues ne sont donc pas la solution au problème de l'inondation, mais elles constituent un véritable problème dans la politique de réduction de la vulnérabilité des biens et surtout des personnes par leur mise en danger. Ainsi, sur la commune de La Faute-surMer, la digue le long de l'estuaire du Lay a-t-elle été surélevée de un mètre dans les mois qui ont suivi la tempête passant de $4 \mathrm{~m}$ à $5 \mathrm{~m}$ NGF. Ces travaux dans l'urgence ont pour objectif de rassurer les populations alors qu'ils ne font qu'accentuer la dangerosité future du secteur à aléa équivalent.

Pour le quatrième critère relatif à la solidité des constructions, il faut ajouter des facteurs aggravant la fragilité des fondations des maisons, notamment pour celles construites dans les dunes ou des accumulations sédimentaires sensibles à l'érosion et l'affouillement des fondations. Par ailleurs, certaines constructions légères (cabanons, mobil-homes, etc.) ont été déplacées avec des hauteurs d'eau de 50 centimètres seulement, provoquant leur destruction. Ces abris sont très nombreux sur les littoraux et représentent un danger extrême pour leurs habitants.

En outre, dans le rapport du 16 septembre 2010 (Pitié et Puech, 2010), en plus des cinq critères analysés ci-dessus, d'autres critères sont évoqués pour le zonage comme : «Une géomorphologie défavorable du site aggravant les effets de submersion (effet de cuvette, absence de cordon littoral naturel), ou rendant impossible l'évacuation d'urgence et l'accès des services de secours; la capacité de protection des habitations, collectivement ou individuellement. » Ces critères n'ont pas été intégrés à la cartographie par les services de l'État.

\section{ÉVOLUTION DES «ZONES NOIRES » À L'ÉCHELLE INFRACOMMUNALE}

La première cartographie des zones à risque concernées par les offres d'acquisitions amiables par l'État a été présentée le 8 avril 2010. Cela signifie que cette cartographie a été réalisée très rapidement, en quelques semaines seulement. Cette rapidité se comprend car il était nécessaire d'apporter des réponses aux habitants concernés et aux assureurs. Cependant, elle explique les vives réactions des populations au moment des réunions publiques et de la nécessité 
d'une révision de la cartographie. Une mission d'expertise complémentaire a donc été diligentée et plusieurs cas de figure ont été rencontrés par les experts. Le premier concerne des habitants ne souhaitant pas vendre leur bien et qui demandaient la sortie de leur maison des zones noires; le deuxième, des personnes dont la maison était située dans la zone de solidarité initiale mais qui en sont sorties avec les nouveaux critères : achetées par l'État, elles seront quand même détruites; le troisième cas de figure des personnes qui au contraire demandaient le classement de leur bien en zone noire pour qu'il soit racheté. Cette dernière catégorie correspond à des personnes qui préfèrent bénéficier de l'indemnisation assurée par le rachat par l'État plutôt que de chercher un acheteur privé sur le marché immobilier.

\section{ÉVOLUTION DE LA CARTOGRAPHIE À TROIS DATES : EXEMPLE DE LA commune de La Faute-sur-Mer}

Trois cartes ont été successivement publiées pour définir les zones noires, le 7 avril, le 16 septembre et le 18 octobre 2010.

Dans le quartier de La Faute-sur-Mer entre le pont du Lay et la pointe d'Arçay, où 29 personnes sont décédées, 608 maisons ont été initialement classées en zone noire. Dans un deuxième temps, ce sont 472 maisons qui ont été proposées à la « délocalisation », 461 dans la zone noire initiale, soit 147 de moins que prévu, et 11 en dehors de la zone initiale. Cependant, le rapport de Pitié et Puech (2011) comptabilise 800 maisons pour ce secteur très bas topographiquement $(9$ hectares sous la cote de $2 \mathrm{~m}$ NGF, 11 hectares entre 2 et $2,5 \mathrm{~m}$

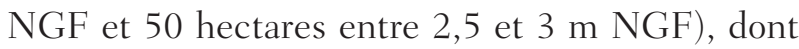
la plus grande partie devrait être considérée comme trop dangereuse pour être conservée (200 de plus que dans la zone de solidarité d'origine).

Les quartiers situés sur la commune de La Fautesur-Mer au nord du pont du Lay ont été inondés avec deux entrées d'eau, par l'ouest suite à la rupture de la dune au lieuidit La Belle Henriette et par l'est suite à la submersion et la rupture de la digue du Lay. Certains quartiers sont à des altitudes inférieures à la cote 2,50 m NGF et présentent un danger important. Ils n'ont pas été classés en zone noire. Le rapport de Pitié et Puech (2011) considère pourtant que 250 maisons dont les planchers d'habitation sont situés sous la cote de 3 m NGF pourraient être qualifiées de dangereuses.

La première cartographie présentait l'avantage d'un zonage homogène sur le plan spatial (figure 6) : c'est la zone de solidarité qui sert encore aujourd'hui de périmètre pour déterminer les acquisitions et les expropriations. Cependant, son tracé pose plusieurs questions : sa délimitation ouest est surprenante puisqu'elle suit parfaitement la route (rue de la pointe d'Arçay), comme si l'inondation s'était cantonnée à un seul côté de la route. De même, au sein du périmètre délimité, certaines maisons avaient été peu ou pas concernées par les inondations : or, elles sont toutes soumises au même régime. Les cartes établis le 16 septembre et le 18 octobre 2010 ont été réalisées en s'appuyant sur de nouveaux critères (les cinq précédemment mentionnés) mais elles restent surprenantes en plusieurs endroits (figure 6). Seuls des éléments de terrain permettent d'éclairer ces choix : la microtopographie semble le plus déterminant (figure 7).

L'État a donc proposé aux habitants situés en zones noires pour les deux départements de Vendée et de Charente-Maritime l'acquisition à l'amiable de leur maison, selon l'article 561-3 du code de l'environnement. En février 2011, sur les 1574 maisons concernées, 1113 ont fait l'objet d'un accord pour une acquisition amiable. Au 2 février 2011, 794 actes de vente ont été signés (325 en CharenteMaritime et 469 en Vendée), pour un montant de 200,5 millions d'euros (MEEDTL, 2011).

\section{DISCUSSION : VERS UNE CARTOGRAPHIE DES ZONES NOIRES POTENTIELLES À L'ÉCHELLE DU LITTORAL FRANÇAIS}

Le rachat des maisons dans les zones noires ne tient pas compte de l'élévation du niveau marin planétaire alors qu'il est demandé de l'inclure dans la révision des PPR. Ce scénario des zones noires post-Xynthia est donc qualifié « d'optimiste » par le rapport de Pitié et Puech (2010).

L'introduction d'un mètre supplémentaire en vue de l'élévation du niveau marin planétaire est discutable. La référence du mètre correspond à la vision pessimiste des expertises du GIEC pour la fin du $\mathrm{XXI}^{\mathrm{e}}$ siècle. Cette décision politique prise en période de post-crise se veut donc a priori prudente. 


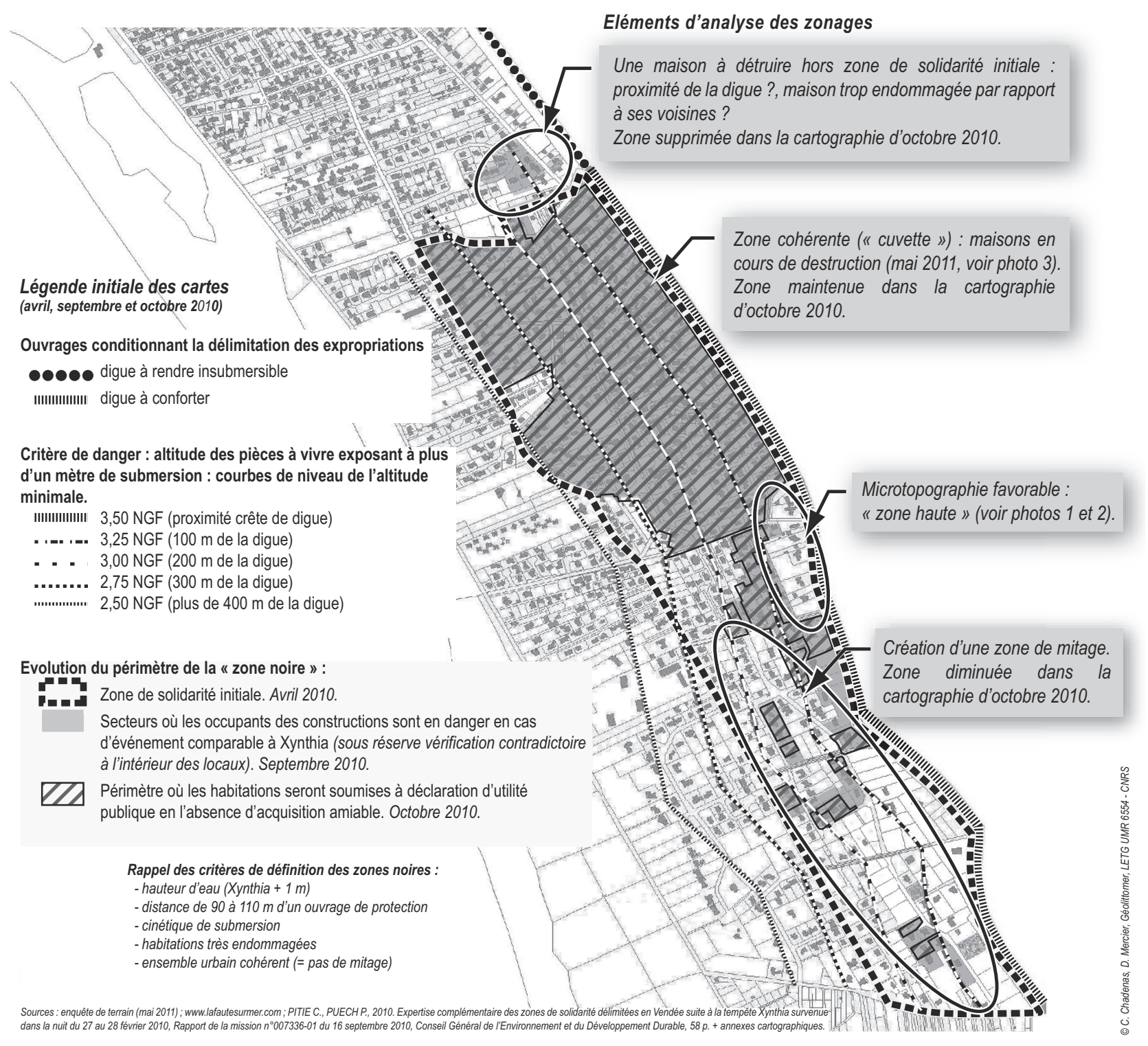

Figure 6 : Périmètre évolutif de la « zone noire » sur la commune de La Faute-sur-Mer

Evolutionary perimeter of the «black zone» on the municipality of La Faute-sur-Mer

Cependant, des valeurs plus importantes sont connues pour l'histoire des littoraux métropolitains. Si l'on veut prendre les plus hautes eaux connues (PHEC) à titre conservatoire, les valeurs extrêmes seraient alors l'altitude des niveaux marins de l'interglaciaire Éémien supérieur de 2 à 4 mètres audessus du zéro actuel. Ce haut niveau marin correspond à une séquence climatique comparable dans l'Arctique à ce qui est pronostiqué dans les modèles climatiques pour la fin du Xxi ${ }^{\mathrm{e}}$ siècle (Solomon et al., 2007). La hausse des températures du bassin arctique induirait alors la fonte partielle de l'inlandsis groenlandais et une forte élévation du niveau marin planétaire. La seule incertitude est de savoir à quel rythme ce processus, connu lors de l'Éémien, est susceptible de se reproduire dans le futur. Alors, la question des 60 centimètres ou du mètre serait largement obsolète.

En plus de ce paramètre d'élévation du niveau marin, il est important de prendre en considération les phénomènes de déformation du plan d'eau à la côte liés au phénomène de déferlement ou wave setup. De plus, les submersions marines peuvent être amplifiées par une dynamique méconnue bapti- 


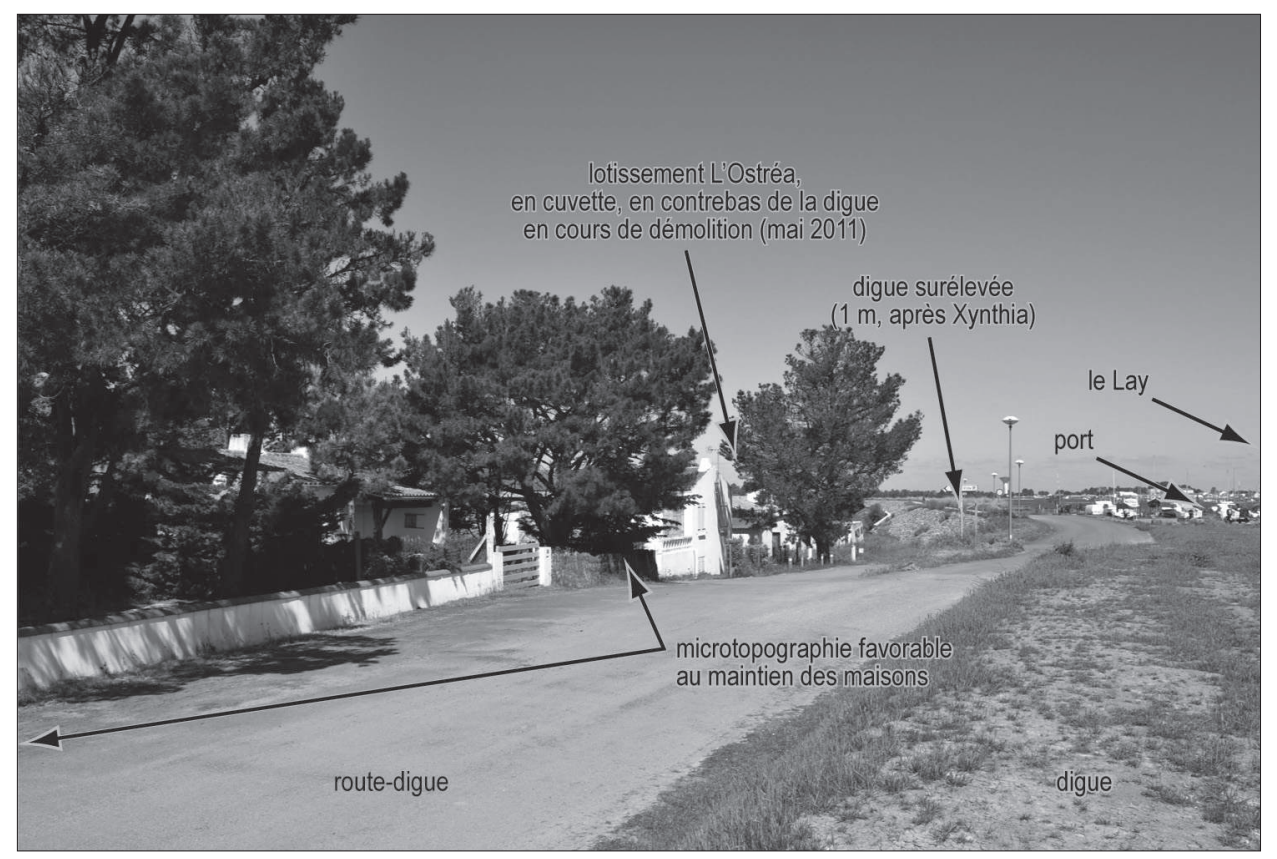

Figure 7 : La microtopographie permet d'expliquer le mitage de l'espace au sein de la zone noire, entre les maisons détruites et les autres (cliché $\mathrm{C}$. Chadenas, 12 mai 2011)

The microtopography allows to explain the mitage of the space within the "black zone", between the destroyed houses and the others. sée seiche côtière. Il s'agit d'oscillations stationnaires générées par des perturbations externes comme les vagues piégées dans des zones plus ou moins confinées, souvent semi-fermées telles que les baies ou les estuaires, comme le Pertuis Breton, et ayant une période propre comprise entre quelques minutes et quelques heures (Pérherin, 2007). Des études ont montré pour deux communes finistériennes qu'il fallait aussi intégrer les tendances érosives des dunes littorales qui peuvent, à plus ou moins long terme, perdre leur fonction protectrice vis-à-vis des enjeux situés en arrière de celles-ci (Suanez et al., 2007; Cariolet et Suanez, 2009; Cariolet, 2010; Cariolet et al., sous presse).

Une étude récente conduite sur l'ensemble du littoral français par les services de l'État estime à 590000 hectares les surfaces situées sous les niveaux marins centennaux et 735500 hectares sous les niveaux marins centennaux $+1 \mathrm{~m}$ (Pérherin et al., 2010). Les régions les plus concernées sont celles des Pays-de-la-Loire, Poitou-Charentes et Nord-Pas-de-Calais. Les régions PACA, Aquitaine et Languedoc-Roussillon sont celles dont les surfaces de zones basses augmentent le plus avec une élévation du niveau marin. Par ailleurs, selon la base de données GASPAR, les régions ayant eu le plus grand nombre d'arrêtés de catastrophes naturelles liées à la mer depuis 1982 sont les régions
Bretagne et Basse-Normandie. Les Alpes-Maritimes et les secteurs estuariens de Gironde et de Seine sont également particulièrement sensibles. Cette même étude recense les enjeux exposés dans ces zones littorales et estime à 265156 le nombre de bâtiments, qu'ils soient administratifs, industriels ou résidentiels, à 16804 kilomètres le linéaire d'infrastructures de transport et enfin à 382640 hectares les surfaces des sites d'intérêt écologique. Cette première approche très instructive doit être approfondie à l'échelle communale et infracommunale en croisant les indicateurs retenus et en les affinant (altimétrie, nombre d'arrêtés de CatNat, présence d'enjeux, etc.). Par exemple, pour le département de la Vendée, les communes de Barbâtre et de la Guérinière sur l'île de Noirmoutier, les communes de Bouin, Saint-Jean-de-Monts et de Saint-Hilairede-Riez possèdent des secteurs particulièrement vulnérables, qui mériteraient des études précises.

Pour les communes de Loire-Atlantique, les services de la direction départementale des territoires et de la mer (DDTM 44) ont retenu la côte de référence de 4,20 m IGN 69 sur l'ensemble du littoral. Les marégraphes de références retenus ont été ceux de Port Navalo, Saint-Nazaire, La pointe de SaintGildas et le Port du Collet. Les niveaux extrêmes enregistrés au moment de Xynthia ont dépassés de 6 à $20 \mathrm{~cm}$ les niveaux marins centennaux calculés 
par le Service Hydrographique et Océanographique de la Marine (SHOM).

Un premier recensement des terrains de campings sur le département de Loire-Atlantique a été réalisé par les services de l'État. Il comptabilise 124 campings situés dans les communes littorales, dont 14 possèdent tout ou une partie de leur terrain sous la cote de référence de 4,20 m avec 7 campings sous la côte de 3,20 m (soit un mètre de submersion potentielle correspondant au premier critère). Un seul camping est situé dans une zone de cinétique rapide. Cependant, aucun secteur n'a été délimité en « zone noire » en Loire-Atlantique, alors que selon les critères appliqués en Vendée et en CharenteMaritime, des secteurs avec plus d'un mètre ont été recensés sur la commune d'Assérac, des habitations à moins de $100 \mathrm{~m}$ d'une digue se trouvent sur les communes de Batz-sur-Mer, La Baule, Le Pouliguen, Corsept, Les Moutiers-en-Retz et Pornic et qu'enfin le secteur du Port du Collet, sur la commune des Moutiers-en-Retz, constitue une zone de submersion violente à forte cinématique.

La tempête Xynthia permet de donner un coup d'accélérateur pour certains points de la politique de prévention qui a pris beaucoup de retard et de les préciser (voir Circulaire du 27 juillet 2011). Météo-France a programmé l'introduction d'une vigilance «vague-submersion ». Il a également été décidé de lancer un "plan national submersions rapides » qui a été validé en Conseil des ministres le 13 juillet 2010. Un plan digue est également lancé. En une année, 190 chantiers ont été menés pour un montant total de travaux de 42,6 millions d'euros. Certaines digues ont été rehaussées comme celle qui longe l'estuaire du Lay le long de la commune de La Faute-sur-Mer alors même que les maisons sont rasées (figure 8; Verger, 2011). Cette ambiguïté dans la politique des digues est surprenante. « Nous voulons renforcer les digues pour améliorer la protection des populations. Mais je veux dire tout de suite que nous ne créerons pas un mètre de plus de digue nouvelle au service de la spéculation immobilière. [...]. Enfin, compte tenu de l'effet des digues sur le reflux des eaux, une précaution élémentaire aurait dû être prise depuis longtemps : dans les zones à risque élevé de submersion, il ne doit plus être possible de construire des logements derrière des digues. Car tôt ou tard, quelle que soit leur hauteur, quelle que soit leur solidité, celles-ci sont un jour submergées. Un peu de bon sens ne nuit pas en matière d'urbanisme. » Extrait du discours du Président de la République française à La Roche-sur-Yon le 16 mars 2010.

La destruction de maisons après une inondation comme en Vendée un an après Xynthia n'est pas une nouveauté en France, puisque des biens immobiliers avaient été détruits à Vaison-la-Romaine suite aux inondations meurtrières du 22 septembre 1992. Par ailleurs, des maisons ont également été détruites sur le littoral normand, à titre préventif cette fois, dans des secteurs où le recul des falaises menaçait la sécurité des biens et des personnes, comme sur la commune de Criel-sur-Mer, à l'est de Dieppe (Meur-Férec et Morel, 2004; Paskoff, 2001). "L'option du "retrait" est une solution extrême. Ce contexte impose aux acteurs de la gestion des risques naturels côtiers la mise en place d'une large concertation avec les populations littorales. Le développement de recherches sur l'intégration de la perception ou de la représentation des risques naturels littoraux dans les stratégies de gestion des risques naturels côtiers devrait contribuer à l'élaboration de ces politiques» (Deboudt, 2010).

Il faut également souligner l'absence sur de nombreux territoires à risque de Plan communal de Sauvegarde, pourtant rendus obligatoires par l'article 13 de la loi n² 2004-811 du 13 août 2004 de modernisation de la sécurité civile. Cet article oblige les communes soumises à un Plan de Prévention des Risques approuvé (risque naturel) ou comprises dans le champ d'application d'un Plan Particulier d'Intervention (risque technologique) à mettre en

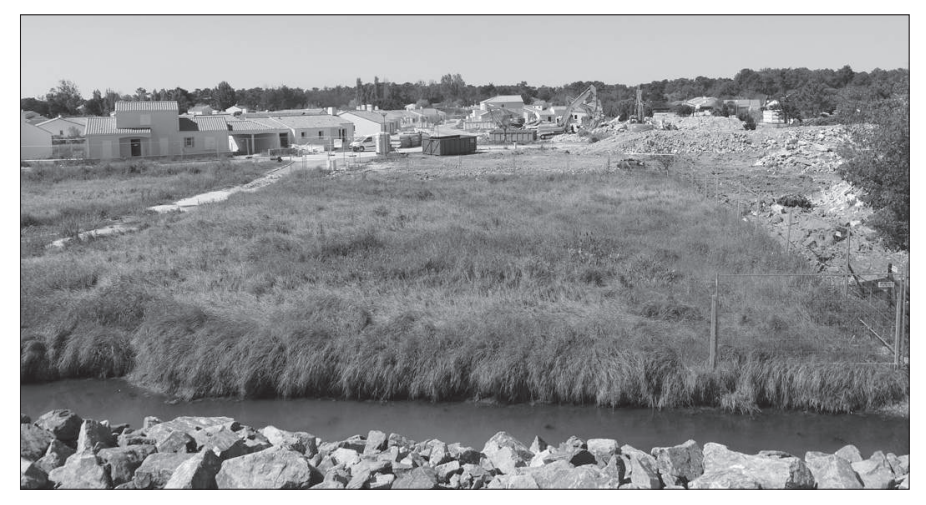

Figure 8 : Maisons détruites sur la commune de La Faute-sur-Mer (cliché D. Mercier, 12 mai 2011)

Houses destroyed on the municipality of La Faute-sur-Mer 
place un Plan Communal de Sauvegarde. Cependant, s'il n'y a pas ou peu de PCS, c'est aussi parce que peu de communes ont adopté des PPR. Sur 864 communes exposées au risque de submersion marine, seulement 46 possèdent un PPR approuvé.

Toutefois, doter une commune d'un PPR n'est pas suffisant pour ne serait-ce que prévenir les habitants du risque. Le rapport du Sénat (Anziani, 2010b) précise en effet le lien insuffisant qui existe entre les PPR et les documents d'urbanisme, car si leur intégration est prévue par la loi, en pratique « l'annexion du PPR au document d'urbanisme, PLU principalement, n'entraîne pas nécessairement de cohérence entre les deux. Il revient donc systématiquement aux services compétents de s'assurer de la compatibilité entre les deux, les dispositions du PPR approuvé prévalant sur le document d'urbanisme en cas de divergence. Et il ne doit pas y avoir ambiguïté pour l'utilisateur ou le particulier qui consulte le document d'urbanisme : les documents graphiques doivent faire état de l'existence du PPR approuvé et renvoyer à celui-ci ». De plus, le PPR n'est pas l'unique outil de planification et d'aménagement du territoire à la disposition des élus. «D'autres techniques foncières qui ne sont pas spécifiques à la submersion marine peuvent être mises en ouvre afin de réguler l'urbanisation du littoral et ainsi réduire la vulnérabilité au risque de submersion marine » (Mulot et al., 2010) : l'article R 561-1 du code de l'environnement (amendement $n^{\circ} 1241$ au projet de loi portant engagement national pour l'environnement) pour l'expropriation des habitations menacées; l'échange de terrains (entre des propriétaires privés et une commune par exemple); le droit de préemption. Ces trois exemples constituent des outils à la disposition des élus pour limiter le risque. Au-delà de ces plans techniques, et comme le soulignaient il y a plus de vingt ans Miossec et Cabanne (1990) : «Il est rare que les documents d'urbanisme aient été mis rapidement en relation avec l'évolution du trait de côte et avec la sensibilité particulière des formes du relief. »

\section{Conclusion}

La tempête Xynthia du 28 février 2010 est révélatrice d'une politique française de gestion des risques naturels largement défaillante. Les zones noires ont été définies par les services de l'État selon des cri- tères difficilement applicables. L'exemple de la commune de La Faute-sur-Mer en Vendée montre que la cartographie des zones noires et la détermination du nombre de maisons à détruire sont délicates à délimiter.

Les critères retenus par les services de l'État pour définir les zones de danger mortel, ou zones noires, correspondent à des secteurs bas topographiquement où la hauteur d'eau est au moins égale à $1 \mathrm{~m}$, situées à moins de $100 \mathrm{~m}$ d'une digue, présentant une cinétique de submersion rapide, où la solidité des habitations est insuffisante et l'ensemble de la zone doit éviter le mitage. Chacun de ces critères est discutable car ils sont parfois insuffisamment protecteurs et de ce fait ne vont pas dans le sens de la mise en sécurité totale des personnes.

La cartographie des zones noires faites a posteriori est difficile à mener à bien comme nous le montre cet exemple récent alors que cette politique devrait s'appliquer a priori pour éviter de nouvelles victimes des inondations et des submersions marines. Cette réflexion doit être menée sur l'ensemble des littoraux français et des zones inondables le long des cours d'eau. Le travail est immense car il doit se faire à l'échelle infracommunale. Les décisions qui en découleront nécessiteront surtout un véritable courage politique. Raser des habitations s'inscrit dans une logique de repli peu développée en France et contraire à la politique technicienne des ingénieurs et des grands corps de l'État, jusqu'ici dominante, de lutte technique contre les éléments (la mer ou les fleuves). Xynthia prouve une fois de plus que cette politique est illusoire, onéreuse et faillible, mettant en danger des vies humaines. Or, les décisions prises rapidement de raser les maisons présentant un risque mortel pour les populations pourraient se comprendre dans une logique qui amorcerait, si elles se perpétuaient dans le temps, un véritable virage stratégique. Les événements du Var de juin 2010 nous prouvent que le concept de «zone noire » ne s'applique plus, même a posteriori. Il est alors légitime de se poser la question de la transcription de cette volonté politique, voire des fondements de celle-ci. En effet, un an avant Xynthia, le même Président appelait dans un discours du 29 avril 2009 sur le Grand Paris, « à sortir du respect passif d'une réglementation de plus en plus pesante... à rendre constructibles les zones inondables pour des bâtiments adaptés à l'environnement et au risque ». 
Cependant, les zones inondables le long des fleuves français et de submersion marine sur les littoraux sont connues depuis longtemps et auraient dû faire l'objet de plan de prévention des risques drastiques interdisant les constructions dans les zones les plus exposées. Le mal est fait. Il importe aujourd'hui de ne plus construire, d'adapter l'habitat existant quand cela est possible et de raser ce qui ne peut garantir la sécurité des personnes pour qu'une politique préventive soit efficace et évite, autant que faire se peut, de nouvelles victimes. Il serait souhaitable que la submersion marine consécutive à la tempête Xynthia amorce un changement majeur dans la politique de prévention des risques en France, comme ce fut le cas en Angleterre et aux Pays-Bas après celle de 1953 (Lumbroso et Vinet, 2011).

\section{Remerciements}

Les auteurs remercient Andrée Dubois, cartographe à l'Institut de Géographie et d'Aménagement Régional de l'Université de Nantes (IGARUN) et Laurent Pourinet, cartographe au laboratoire Géolittomer (CNRS - UMR 6554 LETG), pour avoir dessiné la figure 1. Ils remercient les acteurs des services de l'État et les habitants rencontrés sur le terrain pour les précisions et les échanges constructifs. Les relecteurs Valérie Morel, Philippe Deboudt et Serge Suanez sont également remerciés pour les remarques formulées ayant permis de préciser certains aspects de ce travail.

\section{Bibliographie}

AnZiani A., 2010a. Rapport d'information sur les conséquences de la tempête Xynthia (rapport d'étape), $\mathrm{n}^{\circ}$ 554, SÉNAT, 100 p.

AnZIANi A., 2010b. Xynthia : une culture du risque pour éviter de nouveaux drames (rapport d'informations), $\mathrm{n}^{\circ}$ 647, SÉNAT, $100 \mathrm{p}$.

Cariolet J.-M., 2010. Use of high water marks and eyewitness accounts to delineate flooded coastal areas: The case of Storm Johanna (10 March 2008) in Brittany, France, Ocean E Coastal Management, vol. 53, n 11, p. 679-690.

Cariolet J.-M., Suanez S., 2009. Approche méthodologique pour une cartographie du risque de submersion des côtes basses, La Houille Blanche, $\mathrm{n}^{\circ}$ 2, p. 52-58.

Cariolet J.-M., Suanez S., Postec A., Meur-Férec C., Cartographie du risque de submersion marine. Analyse critique du zonage réglementaire PPR sur la commune de Guissény (Finistère), Cybergeo, n 586, [http://cybergeo. revues.org/25077]; DOI : 10.4000/cybergeo.25077.
Chauveau E., Chadenas C., Comentale B., Pottier P., Blanleil A., Feuillet T., Mercier D., Pourinet L., Rollo N., Tillier I., Trouillet B., 2011 a. Xynthia, premières leçons d'une catastrophe, Cybergeo, n 538, [http:// cybergeo.revues.org/23763].

Chauveau E., Pottier P., Chadenas C., Mercier D., Pourinet L., Feuillet T., Comentale B., Blanleil A., 2011 b. La catastrophe Xynthia : un processus d'urbanisation littorale face à un fait de nature, Les Cahiers Nantais, $n^{\circ} 1$, p. 37-51.

Conseil général de Charente-Maritime, 2010. Rapport d'activité des services de l'État en Charente-Maritime, 9 premiers mois de mesures pour faire face à l'événement et répondre aux dégâts causés, 23 p.

Dion R., 1961. Histoire des levées de la Loire, Paris, 312 p.

Deвoudt P., 2010. Vers la mise en œuvre d'une action collective pour gérer les risques naturels littoraux en France métropolitaine, Cybergeo, $\mathrm{n}^{\circ}$ 491, [http://cybergeo.revues.org/22964].

Douvinet J., Defossez S., Anselle A., Denolle A.-S., 2011. Les maires face aux plans de prévention du risque inondation (PPRI), L'espace géographique, $\mathrm{n}^{\circ}$ 1, p. 31-46.

Jousseaume V., Mercier D., 2009. Évaluer la vulnérabilité architecturale de l'habitat en zone inondable. L'exemple du Val nantais, in Becerra S., Peltier A. (dir.), Risques et environnement: recherches interdisciplinaires sur la vulnérabilité des sociétés, Paris, L’Harmattan, p. 199-214.

Lumbroso D. M., Vinet F., 2011. A comparison of the causes, effects and aftermaths of the coastal flooding of England in 1953 and France 2010, Natural Hazards and Earth System Sciences, vol. 11, n 8, p. 2321-2333.

Meur-Ferec C., Morel V., 2004. L'érosion sur la frange côtière : un exemple de gestion des risques, Natures Sciences Sociétés, vol. 12, n³, p. 263-273.

MEEDTL, 2011 . Xynthia, un an après : des actions fortes pour prévenir les inondations, 17 février 2011,31 p. [www.developpement-durable.gouv.fr].

Miossec A., Cabanne C., 1990. La protection du littoral : une approche méthodologique (deux années d'enquête collective du Certificat de Géographie de la Mer), Les Cahiers Nantais, n³5-36, p. 153-162.

Mulot V., Vigneron A.-L., Lambert-Hadid M.-L., 2010. Le littoral face aux changements climatiques : la gestion des risques de submersion marine, Méditerranée, $\mathrm{n}^{\circ} 115$, p. 131-137.

Paskoff R., 2001. Lélévation du niveau de la mer et les espaces côtiers, Institut Océanographique, Monaco, 190 p.

Perherin C., 2007. Contribution à l'analyse des phénomènes de surcotes et de submersions marines. Secteur du Pertuis Breton (Vendée), École Nationale des Travaux Publics de l'État, Rapport de Stage, 94 p.

Perherin C., Roche A., Trmal C., Roux I., POnS F., Boura C., Devaux E., Desire G., 2010. Vulnérabilité du territoire national aux risques littoraux, Actes des $8^{e}$ JST, Brest (Quartz), 8-9 décembre 2010, 10 p. [www.cetmef.developpement-durable.gouv.fr/].

Pitie C., Puech P., 2010. Expertise complémentaire des zones de solidarité délimitées en Vendée suite à la tempête Xynthia 
survenue dans la nuit du 27 au 28 février 2010, Rapport de la mission $n^{\circ}$ 007336-01 du 16 septembre 2010, Conseil Général de l’Environnement et du Développement Durable, 58 p. + annexes cartographiques.

Seidou O., Marche C., Mahdi T., 2004. Aspects aléatoires de l'érosion d'une digue : simulations de la brèche par des algorithmes génétiques, Revue Canadienne de Génie Civil, vol. 31, n 6, p. 927-942.

Solomon S., Qin S., Manning M., Chen Z., Marquis M., Averyt K. B., Tignor M., Miller H. L. (dir.), 2007. IPCC 2007: Climate change 2007. The physical science basis, Contribution of working group I to the fourth assessment report of the Intergovernmental Panel on Climate Change, Cambridge University Press, 996 p.

Suanez S., Fichaut B., Sparfel L., 2007. Méthode d'évaluation du risque de submersion des côtes basses appliquée à la plage du Vougot, Guissény (Bretagne), Géomorphologie : Relief, Processus, Environnement, n 4, p. 319-334.
Verger F., 2011. Digues et polders littoraux : réflexions après la tempête Xynthia, Physio-Géo, vol. 5, [http://physio-geo. revues.org/1740].

Vinet F., 2010. Le risque inondation. Diagnostic et gestion, Paris, Lavoisier, 318 p.

Vinet F., Defossez S., Leclere J.-R., 201 la. Comment se construit une catastrophe, in Mercier D., Acerra M. (dir.), Xynthia, une tragédie prévisible, Place Publique, Hors série, p. 9-16.

Vinet F., Boissier L., Defossez S., 201 lb. La mortalité comme expression de la vulnérabilité humaine face aux catastrophes naturelles: deux inondations récentes en France (Xynthia, Var, 2010), VertigO, vol. 11, n², [http://vertigo. revues.org/11074].

Zerrouk N.-E., Marche C., 2005. Une contribution expérimentale à l'étude de l'érosion d'une digue par submersion et son analyse, Revue des Sciences de l'Eau, vol. 18, n³, p. 361-401. 